\title{
Ocorrência de rinite atrófica e pneumonia em suínos híbridos e sem raça definida em criação intensiva
}

\author{
Juliana Fortes Vilarinho Braga ${ }^{*}$, Maurício de Paula Ferreira Teixeira', João Batista Lopes², \\ Manoel Henrique Klein Júnior², Silvana Maria Medeiros de Sousa Silva² \\ 'Universidade Federal de Minas Gerais, Belo Horizonte, MG, Brasil \\ 2 Universidade Federal do Piauí, Teresina, PI, Brasil \\ *Autor correspondente, e-mail: jufortes22@gmail.com
}

\section{Resumo}

A rinite atrófica (RA) e as pneumonias estão entre as doenças respiratórias mais frequentemente diagnosticadas nos sistemas de produção de suínos, aos quais causam prejuízos econômicos significativos. Objetivou-se determinar a ocorrência e o grau de RA e pneumonias em rebanhos de suínos híbridos e sem raça definida de criações intensivas, além de verificar se há associação entre a ocorrência dessas enfermidades nos rebanhos estudados. Para isso, foram estudados 30 suínos híbridos (Grupo I) e 30 suínos sem raça definida (Grupo II) de criação intensiva. O Índice de Rinite Atrófica (IRA) dos rebanhos dos grupos I e II foi, respectivamente, 1,00 e 0,57. No grupo I, 20,8\% (5/24) dos suínos apresentaram pneumonia, enquanto nenhum dos animais do grupo II exibiu esta patologia. Não houve correlação entre presença de rinite atrófica e pneumonia nos animais deste estudo. Estes são os primeiros dados sobre a ocorrência destas enfermidades em suínos no Estado do Piauí e demonstram que as doenças estão presentes em animais de criação intensivas, com ocorrência variando com o tipo racial, sendo os suínos híbridos mais afetados por ambas.

Palavras-chave: suinocultura, doenças respiratórias, Nordeste, suídeos

\section{Atrophic rhinitis and pneumonia occurence in hybrid and undefined breed swines from intensive production system}

\begin{abstract}
The atrophic rhinitis (AR) and pneumonia are among the most frequently diagnosed respiratory diseases in swine production systems and can lead to significant economic losses. The aim of this study was to determine the occurrence and degree of AR and pneumonia in hybrids and undefined breed swine herds submitted to intensive farming system and check if there is an association between the occurrence of these diseases in the herds. For this, we used 30 swines hybrids (Group I) and 30 undefined breed swines (Group II) from intensive farming system. The index of atrophic rhinitis (IAR) of herds for Groups I and II were, respectively, 1.00 and 0.57. In Group I, 20.8\% (5/24) of the pigs presented pneumonia, while none of the animals in Group II exhibited this pathology. There was no correlation between the presence of atrophic rhinitis and pneumonia for the studied animals. These are the first data on the occurrence of these diseases in swines of Piauí State, Brazil. We demonstrated that these pathologies are present in animals from intensive farming systems and that occurrence varied according to racial type, being the hybrid swines the most affected animals by AR and pneumonia.
\end{abstract}

Keywords: swine culture, respiratory diseases, Northeast of Brazil, suidae 


\section{Introdução}

A suinocultura moderna, baseada na criação intensiva e sistemática dos animais, gera indiscutiveis ganhos de produtividade em escala. Entretanto, a grande concentração de animais facilita a disseminação de doenças infecciosas, comprometendo a sanidade e desempenho produtivo dos animais e gerando prejuízos ao agronegócio (Toledano, 2008).

Dentre os problemas sanitários que ocorrem nos rebanhos, as doenças respiratórias ocupam lugar de destaque por sua ampla distribuição geográfica, frequência e intensidade com que atingem os sistemas de produção (Sobestiansky et al., 2001).

A rinite atrófica (RA) dos suínos é uma enfermidade infectocontagiosa frequentemente diagnosticada nos sistemas convencionais de produção de suínos (Piffer \& Brito, 1993) e caracterizada por atrofia dos cornetos nasais e, em casos mais graves, deformação do focinho (Martins et al., 1985). Embora a doença seja considerada de etiologia multifatorial (Sobestiansky et al., 2001), pode ser reproduzida utilizando somente as toxinas dermonecróticas recombinantes da bactéria Pasteurella multocida tipo A ou D, sendo o desenvolvimento das lesões favorecido por infecção concomitante com a bactéria Bordetella bronchiseptica (Santos \& Guedes, 2010). Ambos os agentes possuem amostras designadas TDN+ (toxina dermonecrótica) devido à sua capacidade produzir uma toxina que provoca necrose, sendo as amostras de $B$. bronchiseptica TDN+ amplamente distribuídos em rebanhos suínos (Brito et al., 1993).

Embora haja divergência sobre a predisposição de animais com RA para adquirir pneumonias, foi observado que estas podem ocorrer em animais com RA grave (Brito et al., 1993). Isso ocorre porque B. bronchiseptica, após aderir às células epiteliais nasais e traqueais, promove estase ciliar e aumento de secreção de muco, favorecendo a colonização desse epitélio por patógenos como a P. multocida (Dugal et al., 1992). Além de Pasteurella multocida, as bactérias Mycoplasma hyopneumoniae e Actinobacillus pleuropneumoniae também são considerados os mais importantes patógenos envolvidos nos quadros pneumônicos em criações intensivas de suínos no país (Piffer \& Brito, 1993). As pneumonias produzidas por P. multocida e M. hyopneumoniae são caracterizadas por áreas de consolidação ou hepatização cranioventrais, de coloração vermelho-escura ou acinzentada, localizada nas porções cranioventrais, sempre seguindo a orientação lobular (Santos \& Guedes, 2010).

Sabe-se que enfermidades como rinite atrófica, pneumonia micoplásmica e a pleuropneumonia ocorrem de forma endêmica no nosso país e provocam prejuízos econômicos consideráveis à produção suinícola (Piffer \& Brito, 1993). Entretanto, no estado do Piauí, quinto maior produtor de suínos em número de cabeças na região nordeste do país (Brasil, 2012), estudos sobre a ocorrência destas enfermidades na população suídea não se encontram disponíveis na literatura. Diante disso, objetivouse determinar a ocorrência e o grau de rinite atrófica e pneumonias em suínos híbridos e sem raça definida de criações intensivas no estado do Piauí, além de verificar a associação entre essas patologias.

\section{Material e Métodos}

Foram analisadas as conchas nasais de 60 suínos, sendo 30 animais híbridos, mestiços de Landrace, Large White e Duroc (Grupo I - Figura 1A) e 30 animais sem raça definida ou caipiras (Grupo II - Figura 1B). Os suínos do grupo I foram provenientes de duas propriedades no município de Teresina, Piauí, enquanto os animais do grupo Il foram oriundos de apenas uma delas. Todos os suínos foram criados sob regime intensivo, alimentados com ração comercial duas vezes ao dia e abatidos aos seis meses de idade, pesando aproximadamente $90 \mathrm{~kg}$ (híbridos) e $30 \mathrm{~kg}$ (sem raça definida). A criação não possuía programa vacinal específico, sendo assim os animais não receberam qualquer vacinação.

Após o abate dos animais, procedeuse a secção transversal do focinho, entre $01^{\circ}$ e $2^{\circ}$ dentes pré-molares para visualização das conchas nasais e sua posterior classificação. A avaliação das conchas nasais foi feita por apreciação visual, como descrito por Martins et al. (1985), classificando-se como 
normais (pontuação zero); pequeno desvio da normalidade (pontuação 1); atrofia definida (pontuação 2); e atrofia grave ou completa (pontuação 3). Conhecidos os graus de lesão dos cornetos nasais de cada animal, calculouse $o$ índice de rinite atrófica (IRA) dos rebanhos dos grupos I e ll, o qual é baseado na média ponderada da graduação (pontuação) das alterações das conchas nasais (Brito et al., 1990).
Para determinar a ocorrência de pneumonia, foram avaliados macroscopicamente os pulmões de 48 suínos (24 de cada grupo), considerando a consistência, coloração e aspectos das lesões. Para verificar a associação entre RA e pneumonia foram realizadas análises estatísticas utilizando-se o teste Qui-quadrado com níveis de probabilidade de $1 \%$ e $5 \%$.

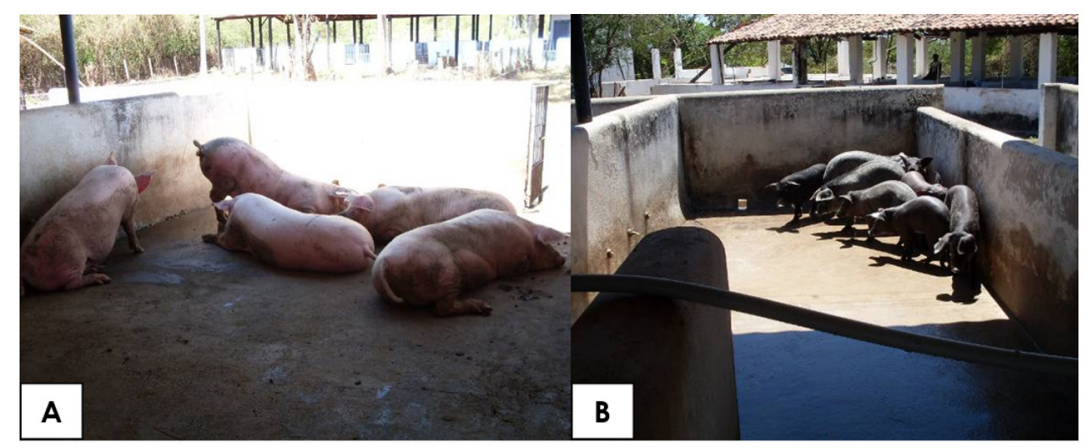

Figura 1. Suínos híbridos pertencentes ao Grupo I (A) e suínos sem raça definida (B) do Grupo II.

\section{Resultados e Discussão}

A ocorrência da RA nos suínos, independentemente do tipo racial, foi $61,7 \%$ (37/60). A maioria dos animais estudados, 48,3\% (29/60), apresentaram Grau 1 de lesão nas conchas nasais, enquanto 38,3\% (23/60) dos suínos não apresentaram alteração, sendo classificados como Grau zero. Dez por cento (6/60) dos animais possuíam atrofia moderada das conchas, correspondendo ao Grau 2 e $3,4 \%$ (2/60) dos animais apresentaram Grau 3, com lesões severas nas conchas nasais e desvio de septo nasal. A partir desses dados, O IRA do rebanho foi de 0,78 , demonstrando que a rinite atrófica é um problema nesta criação. Este índice foi superior ao observado por Brito et al. (1990) que, estudando rebanhos de Santa Catarina, obtiveram IRA médio de 0,67, enquanto Filippsen et al. (2001) não observaram lesões de atrofia dos cornetos em 80 leitões criados ao ar livre no Estado do Paraná. Esta diferença na ocorrência da doença entre os estudos pode estar relacionada ao sistema de criação dos suínos, uma vez que os suínos estudados foram criados em regime intensivo, no qual há maior densidade populacional, favorecendo o aumento na concentração de irritantes atmosféricos (como amônia, gás sulfídrico e poeira) e predispondo os animais a infecções respiratórias (Sobestiansky et al., 1999).

Analisando isoladamente os suínos do grupo I, a ocorrência da doença foi de 73,3\% (22/30). A maioria dos animais, 53,3\% (16/30), exibiu conchas nasais com leve desvio da normalidade, sendo classificados como Grau 1 (Figuras 2 e 3). Animais com conchas nasais normais, Grau 0, representaram a segunda maior frequência, 26,7\% (8/30), enquanto 13,3\% (4/30) dos suínos apresentaram Grau 2 e 6,7\% (2/30) apresentaram Grau 3, com destruição severa das conchas nasais e desvio de septo nasal. O IRA do Grupo I, calculado à semelhança do rebanho geral, foi igual a 1,00, indicando que a doença causa prejuízos ao rebanho, como ocorre em $73 \%$ dos rebanhos estudados em Santa Catarina, com IRA igual ou superior a 0,46 (Brito et al., 1990) e no sul e sudeste do país, com IRA médio igual a 1,22 qualquer alteração (Talamini et al., 1991). O IRA observado neste Grupo caracteriza os rebanhos onde a rinite atrófica é um problema, justificando-se, neste caso, a adoção de medidas que visem o controle da doença, a qual já prejudica a eficiência do rebanho (IRA > 0,45).

Em relação aos suínos do Grupo II, a ocorrência da RA foi de 50\% (15/30). A maioria dos animais 50,0\% (15/30) não apresentou alterações nas conchas nasais, enquanto 
43,3\% (13/30) revelaram Grau 1 e 6,7\% (2/30) dos suínos apresentaram Grau 2 (Figuras 2 e 4). Não houve animais exibindo lesão de Grau 3. 0 Grupo II teve o IRA de 0,57, demonstrando que também já sofre perdas significativas, com IRA superior a 0,46, como descreve Brito et al. (1993), e necessita de medidas de controle para a doença. Na literatura consultada não há registros de trabalhos com rinite atrófica em suínos sem raça definida, sendo estes os primeiros dados abordando o assunto.

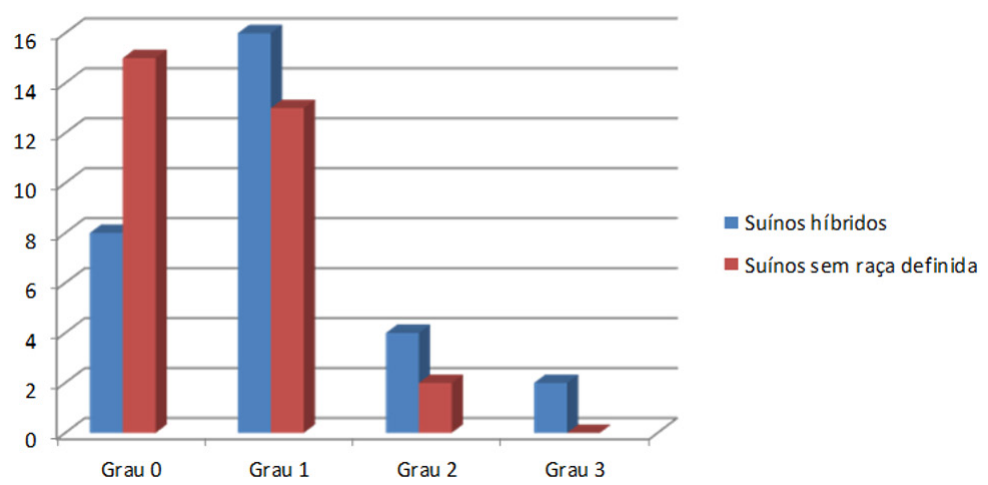

Figura 2. Graus de rinite atrófica em rebanhos de suínos híbridos e sem raça definida em criações intensivas.

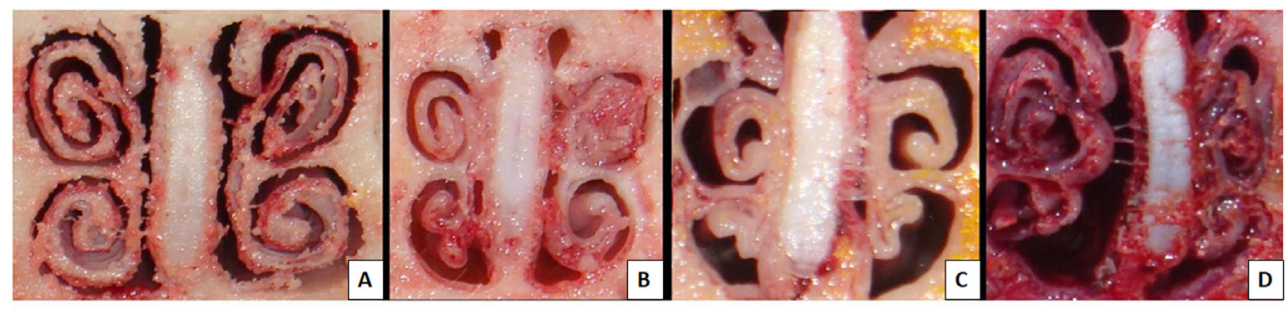

Figura 3. Graus de rinite atrófica em suínos híbridos de criações intensivas do estado do Piauí. (A) Grau 0; (B) Grau 1; (C) Grau 2; e (D) Grau 3.

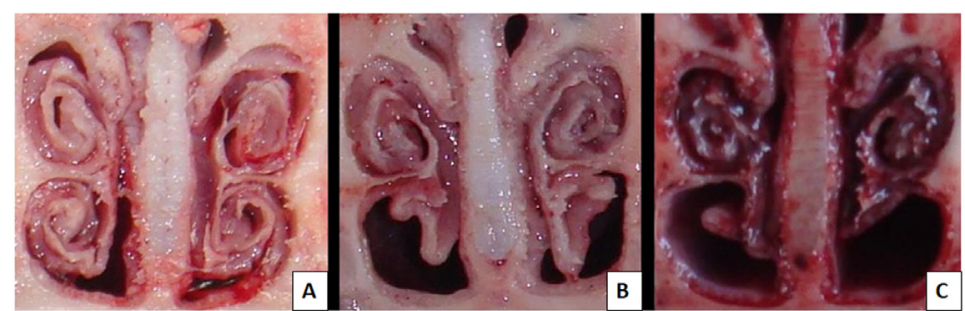

Figura 4. Graus de rinite atrófica em suínos sem raça definida de criações intensivas. A) Grau 0; (B) Grau 1; e (C) Grau 2.

A avaliação macroscópica dos pulmões dos suínos do Grupo I revelou que 20,8\% (5/24) apresentaram broncopneumonia lobular. Essa caracterizou-se por consolidação e coloração vermelho-escuro de lobos craniais, acessório e, ocasionalmente, o terço ventral dos lobos caudais (Figura 5). Dos suínos afetados, $40 \%$ $(2 / 5)$ apresentaram Grau 0 de rinite atrófica e $40 \%$ (2/5) Grau 1, enquanto apenas 20\% (1/5) apresentou Grau 2, revelando atrofia moderada das conchas. Neste estudo não foi observada associação entre pneumonia e rinite atrófica $(p<0,05)$, como observado por Donkó et al. (2005). Brito et al. (1993) acrescentam que a presença de pneumonia existe com maior frequência nos graus mais severos de RA. Entretanto, Sobestiansky et al. (2001) estudando granjas produtoras de terminados tecnificados nos Estados de Santa Catarina, Rio Grande do Sul e Paraná, diagnosticou pneumonia em 54,9\% dos suínos e RA em 49,4\%, porém os autores não correlacionaram a presença das doenças. Resultado semelhante foi visto por Filippsen et al. (2001), estudando a prevalência de doenças 
infecciosas em rebanho de suínos criados ao ar livre na região sudoeste do Paraná, observaram que nenhum animal apresentou lesão de atrofia dos cornetos nasais, porém 12,5\% apresentaram alguma área de consolidação pulmonar.

Apesar de alguns suínos sem raça definida terem exibido lesões de RA, em nenhum desses animais foi constatada pneumonia. Embora a menor ocorrência de pneumonias neste grupo possa ter sido influenciada pelo IRA do rebanho $(0,57)$, inferior ao dos suínos híbridos $(1,00)$, é importante ressaltar que a rusticidade dos animais sem raça definida, ou caipiras, pode ter contribuído para esta observação, uma vez que esses apresentam maior resistência imunológica do que aqueles geneticamente melhorados.

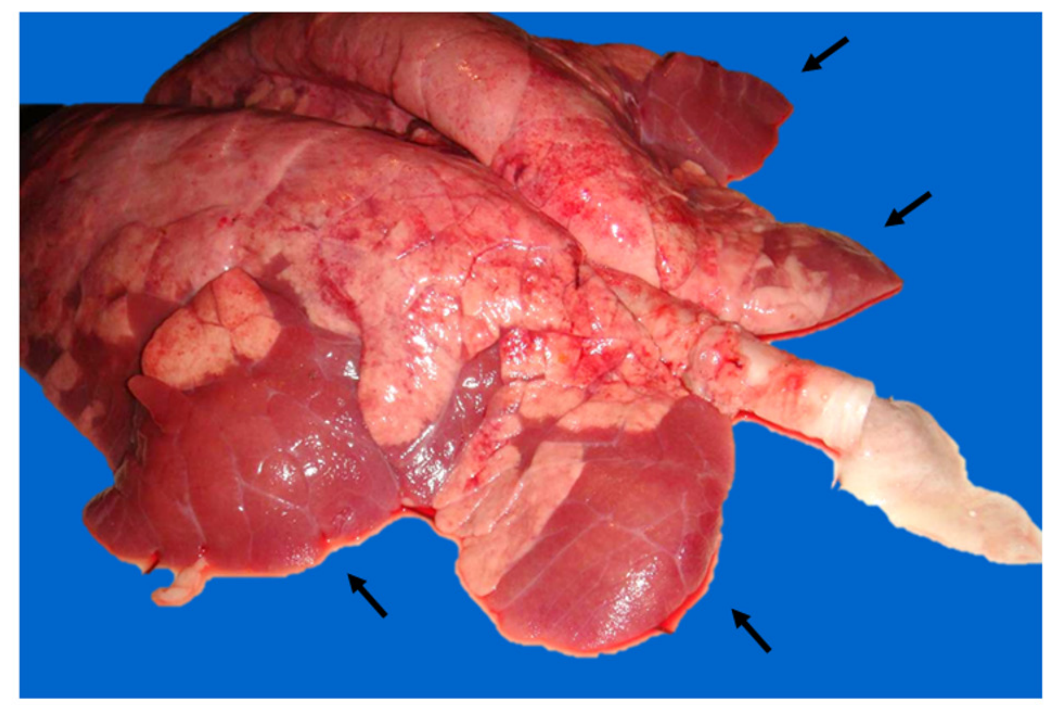

Figura 5. Áreas de consolidação pulmonar cranioventral (setas) indicando broncopneumonia em suíno híbrido (Grupo l) de criação intensiva.

\section{Conclusões}

Estes são os primeiros dados sobre a ocorrência de RA e pneumonia em suínos hibridos e sem raça definida de criações intensivas no estado do Piauí. As observações deste estudo demonstram que a RA afeta suínos independentemente do tipo racial, com ocorrência superior em animais híbridos, estes os únicos em que foi diagnostica pneumonia. Entretanto, não foi observada associação entre as doenças nas criações estudadas. Uma vez que RA e pneumonia afetem suínos do Estado e são sabidamente responsáveis por perdas econômicas significativas nesta espécie, tornase evidente a necessidade de estudos que confirmem causa primária e agentes etiológicos envolvidos, possibilitando a adoção de medidas de controle adequadas às doenças.

\section{Referências}

Brasil. Ministério do Planejamento, Orçamento e Gestão. Instituto Brasileiro de Geografia e Estatística. Indicadores, Agropecuária, Produção Pecuária. 2012. http://www.ibge.gov.br/ home/estatistica/indicadores/agropecuaria/ producaoagropecuaria/default.shtm <Acesso em 12 Fev. 2013>

Brito, J.R.F., Piffer, I.A., Brito M.A.V.P. 1993. Rinite atrófica dos suínos. Suinocultura Dinâmica, 7: 1-5.

Brito, J.R.F., Piffer, I.A., Brito, M.A.V.P., Sobestiansky, J. 1990. Formulação de um índice (IRA) para aplicação na caracterização e classificação de rebanhos com rinite atrófica. Comunicado Técnico - CNPSA 160: 1-4.

Coelho, A.C., Vieira-Brito, F.J., Vieira-Brito, M.G., Rodrigues, E.J. 2004. Pleuropneumonia suína causada por Actinobacillus pleuropneumoniae - diagnóstico e estratégias de controlo. Revista Portuguesa de Ciências Veterinárias 99: 193-198.

Davies, R.L., Maccorquodale, R., Baillie, S., Caffrey, B. 2003. Characterization and comparison of Pasteurella multocida strains associated with porcine pneumonia and atrophic rhinitis. Journal of Medical Microbiology 52: 59-67.

Donkó, T., Kovács, M., Magyar, T. 2005. Association of growth performance with atrophic rhinitis and pneumonia detected at slaughter in a conventional pig herd in Hungary. Acta Veterinaria Hungara 53: 287-298. 
Dugal, F., Belanger, M., Jacques, M. 1992. Enhanced adherence of Pasteurella multocida to porcine tracheal rings preinfected with Bordetella bronchiseptica. Canadian Journal of Veterinarian Research 56: 260-264.

Filippsen, L.F., Leite, D.M.G., Silva, A., Vargas, G.A. 2001. Prevalência de doenças infecciosas em rebanho de suínos criados ao ar livre na região sudoeste do Paraná, Brasil. Ciência Rural 31: 299302.

Martins, E., Scarsi, R.M., Piffer, I.A. 1985. Classificação macroscópica dos graus de atrofia dos cornetos na rinite atrófica dos suínos. Comunicado Técnico - CNPSA 93: 1-3.

Piffer, I.A., Brito, J.R.F. 1993. Pneumonia em suínos. Suinocultura Dinâmica 8: 1-6.

Santos, R.L., Guedes, R.M.C. 2010. Sistema Respiratório. In: Santos, R.L., Alessi, A.C. (ed.) Patologia Veterinária. Roca, São Paulo, Brasil. p.1-50.

Sobestiansky, J., Barcellos, D., Mores, N., Carvalho, L.F., Oliveira, S. 1999. Clínica e Patología Suína p. 374-378.

Sobestiansky, J., Costa, O.A.D., Morés, N., Barioni Jr., W., Piffer, I.A., Guzzo, R. 2001. Estudos ecopatológicos das doenças respiratórias dos suínos: prevalência e impacto econômico em sistemas de produção dos estados de Santa Catarina, Rio Grande do Sul e Paraná. Comunicado Técnico - CNPSA 287: 1-6.

Talamini, D.J.D., Piffer, I.A., Brito, M.A.V.P. 1991. Perdas econômicas decorrentes de diferentes graus de severidade de rinite atrófica em suínos. Comunicado Técnico - CNPSA 169: 1-5.

Toledano, F. Portal Dia de Campo (Sanidade Animal). 2013. http://www.diadecampo. com.br/zpublisher/materias/Materia. asp? $i d=21533 \&$ secao $=$ Sanidade\%20Animal <Acesso em: 12 Fev. 2013>

Vaz, C.S.L., Silva, S.C. 2004. Aspectos recentes da patogênese e diagnóstico da pleuropneumonia suína. Ciência Rural 34: 635-643. 